\title{
ISOTOPIES OF GENERIC PLANE CURVES
}

\author{
by J. W. BRUCE
}

(Received 28 April, 1982)

The aim of this paper is to explore some facets of the geometry of generic isotopies of plane curves. Our major tool will be the paper of Arnol'd [1] on the evolution of wavefronts. The sort of questions one can ask are: in a generic isotopy of a plane curve how are vertices created and destroyed? How does the dual evolve? How can the Gauss map change? In attempting to answer these questions we are taking advantage of the fact that these phenomena are all naturally associated with singularities of type $A_{k}$. Now the bifurcation set of an $A_{k+1}$ singularity and the discriminant set of an $A_{k}$ singularity coincide. So we can apply Arnol'd's results on one parameter families of Legendre (discriminant) singularities (e.g. the duals) to get information on one parameter families of Lagrange (bifurcation) singularities (e.g. the evolutes). For bifurcation sets of functions with singularities other than those of type $A_{k}$ one runs up against problems with smooth moduli-see [4].

The paper is divided into four sections. The first reviews the results of Arnol'd we shall need. In the second we consider the geometry of plane and space curves associated with the families of distance squared and height functions. We determine the conditions under which an isotopy gives rise to generic changes in this geometry. In the third section we prove a transversality result which shows that generic isotopies are open and dense; in particular two connected generic plane curves can be joined by a generic isotopy.

In the final section we describe the generic changes which do occur. The same methods suffice (with suitable modifications) to describe the geometry of generic isotopies of space curves and we hope to do this in a later paper.

The author would like to thank Dr. P. J. Giblin for pointing out a mistake in an earlier version of this paper, and the referee for some very useful comments.

1. In this section we introduce some results of Arnol'd [1] concerning functions defined on the bifurcation and discriminant sets of $A_{k}$ singularities.

A function germ $g: \mathbf{R}, 0 \rightarrow \mathbf{R}, 0$ is said to be of type $A_{k}$ if it can be reduced by a change of co-ordinates in the source to the normal form $f(t)= \pm t^{k+1}$ i.e. if the germs $f$ and $g$ are right equivalent. (Using Taylor's theorem it is not hard to see that $g$ is of type $A_{k}$ if and only if $g^{(p)}(0)=0$, for $1 \leq p \leq k$ and $g^{(k+1)}(0) \neq 0$.) Sometimes the zeros of the function $g$, and nearby functions, will be important and we then simply refer to $g$ as a function. In other context however the values of $g$, and nearby functions, are not relevant, but its critical points are. We then refer to $g$ as a potential function. (We are considering the same germ: there is simply a different emphasis.)

A family of functions $G: \mathbf{R} \times \mathbf{R}^{n}, 0 \rightarrow \mathbf{R}, 0$ is said to be an unfolding of $g$ if the germs $g$ and $G(-, 0): \mathbf{R}, 0 \rightarrow \mathbf{R}, 0$ coincide. The unfolding $G$ is of dimension $n$. The family $G$ is a versal unfolding of the function $g$ with an $A_{k}$ singularity if and only if the truncated Taylor

Glasgow Math. J. 24 (1983) 195-206. 
expansions of $\frac{\partial G}{\partial a_{i}}(t, 0), 1 \leq i \leq n$, span the space of polynomials in $t$ of degree at most $k-1$. (We write this, rather loosely, as $\frac{\partial G}{\partial a_{i}}(t, 0), 1 \leq i \leq n, \operatorname{span} \mathbf{R}[t] /\left\langle t^{k}\right\rangle$. Roughly speaking a versal unfolding of $g$ contains all germs close to $g$. See [2].)

Given an unfolding $G$ of the function $g$ the discriminant set of $G$ is the set germ

$$
D(G), 0=\left\{a \in \mathbf{R}^{n}: G(t, a)=\frac{\partial G}{\partial t}(t, a)=0 \text { for some } t\right\}, 0 .
$$

If $G$ is an unfolding of the potential function $g$ the bifurcation set of $G$ is the set germ

$$
B(G), 0=\left\{a \in \mathbf{R}^{n}: \frac{\partial G}{\partial t}(t, a)=\frac{\partial^{2} G}{\partial t^{2}}(t, a)=0 \text { for some } t\right\}, 0
$$

The first result we require is the following.

THEOREM 1.1 [2, p. 123]. Let $g_{0}$ and $g_{1}$ be right equivalent functions, and $G_{0}$ and $G_{1}$ versal unfoldings of $g_{0}$ and $g_{1}$ respectively, of the same dimension. Then the discriminant sets of $G_{0}$ and $G_{1}$ are diffeomorphic (i.e. there is a germ of a diffeomorphism $\phi: \mathbf{R}^{n}, 0 \rightarrow$ $\mathbf{R}^{n}, 0$ taking $D\left(G_{0}\right), 0$ to $\left.D\left(G_{1}\right), 0\right)$. Similarly if $g_{0}$ and $g_{1}$ are right equivalent potential functions, and $G_{0}$ and $G_{1}$ are versal unfoldings of $g_{0}$ and $g_{1}$ then the bifurcation sets of $G_{0}$ and $G_{1}$ are diffeomorphic.

For the $A_{k}$ singularities $\pm t^{k+1}$ we have the following standard versal (resp. potential versal) unfolding of minimal dimension:

$$
\begin{aligned}
\tilde{F}(x, a) & = \pm t^{k+1}+b_{1} t^{k-1}+\cdots+b_{k-1} t+b_{k} \\
(\text { resp. } F(x, a) & \left.= \pm t^{k+1}+b_{1} t^{k-1}+\cdots+b_{k-1} t\right)
\end{aligned}
$$

By Theorem 1.1 the discriminant (resp. bifurcation) set of any versal unfolding of an $A_{k}$ singularity is diffeomorphic to the product of that of $\tilde{F}$ (resp. $F$ ) with some Euclidean space. (This uses the fact that one can increase the dimensions of the versal unfoldings $\tilde{F}, F$ simply by adding variables to the left of the above expressions which do not appear on the right.) Clearly the discriminant set of an $n$-dimensional versal unfolding of an $A_{k}$ singularity, and the bifurcation set of an $n$-dimensional versal unfolding of an $A_{k+1}$ (potential type) singularity are diffeomorphic.

We now outline the results we need from Arnol'd. In [1] Arnol'd classified generic functions on the discriminant sets of unfoldings of simple singularities, and hence in particular on the discriminant and bifurcation sets of $A_{k}$ singularities. The results will be used by us in the following situation. We shall be considering a versal unfolding $G(t, a, u)$ of the potential function $G(t, 0,0)=g(t)$, where $(a, u)$ are the unfolding parameters and $u$ is a (single) distinguished parameter. The bifurcation (or parent bifurcation) set is defined as above and is a subset of the $(a, u)$-space $A \times U$. The discriminant set arises in the guise 
of the set of critical values of $G$. So we consider the extended unfolding $\tilde{G}(t, a, u, c)=$ $G(t, a, u)-c$. The discriminant (or parent discriminant) set of $\tilde{G}$ is clearly the set

$$
\left\{(a, u, c): G(t, a, u)=c, \frac{\partial G}{\partial t}(t, a, u)=0 \text { for some } t\right\}
$$

The unfoldings $G$ and $\tilde{G}$ give rise to families of bifurcation, resp. discriminant, sets obtained by fixing the parameter $u$. (This explains the parental role of $G$ and $\tilde{G}$.) So we have natural projections $\pi: A \times U \rightarrow U$ (resp. $\pi_{1}: A \times U \times \mathbf{R} \rightarrow U$ ) and we want to consider the restriction to $B(G)$ (resp. $D(\tilde{G})$ ). The results we require follow from the following interpretation of Arnol'd's theorems.

THEOREM 1.2. Let $G(t, a, u)$ be as above. If $1, \frac{\partial G}{\partial a_{i}}(t, 0,0)(1 \leq i \leq n)$ and $\frac{\partial G}{\partial u}(t, 0,0)$ span $\mathbf{R}[t] /\left\langle t^{k}\right\rangle$ then $\tilde{G}$ (resp. $G$ ) is a versal unfolding of the function (resp. potential function) $g(t)$. When this is the case we have the following.

(a) If $1, \frac{\partial G}{\partial a_{i}}(t, 0,0)$ span $\mathbf{R}[t] /\left\langle t^{k}\right\rangle$ the projection $\pi$ (resp. $\pi_{1}$ ) is equivalent, via a bifurcation (resp. discriminant) preserving diffeomorphism, to the trivial projection onto one factor of a product bifurcation (resp. discriminant) set.

(b) If $G$ is of minimal dimension $k-1$ and $1, \frac{\partial G}{\partial a_{1}}(t, 0,0)$ span $\mathbf{R}[t] /\left\langle t^{k-1}\right\rangle$ then the projection $\pi_{1}$ (resp. $\pi$ ) is equivalent to the projection of the standard discriminant (resp. bifurcation) set of $\tilde{F}$ (resp. $F$ ) above onto the $a_{1}$-coordinate.

Proof. The first assertion follows immediately from our definition of versality. Parts (a) and (b) follow from (4.5) of [1]. (Roughly speaking (a) follows because the discriminant (or bifurcation) set must be a product in the $u$-direction since $g$ is versally unfolded by the a parameters). To deduce (b) from [1] we have to show that the condition given above coincides with Arnol'd's criterion for a generic function, namely that if $f$ is the function in question on the standard discriminant set of $\tilde{F}$ then $\frac{\partial f}{\partial b_{1}}(0)$ must be non-zero. (We shall discuss the bifurcation set case later.) To simplify notation in what follows we will relabel $c$ as $a_{k-1}$ and replace $A \times \mathbf{R}$ by $A$.

Using the fact that $\tilde{G}$ is a versal unfolding of an $A_{k}$ singularity of minimal dimension we can find smooth germs $\phi: \mathbf{R} \times \mathbf{R}^{k}, 0 \rightarrow \mathbf{R}, 0, \psi: \mathbf{R}^{k}, 0 \rightarrow A \times U, 0$ with $\phi(-, 0): \mathbf{R}, 0 \rightarrow \mathbf{R}$, 0 a diffeomorphism, $\psi$ a diffeomorphism and

$$
\tilde{G}(\phi(t, b), \psi(b))=t^{k+1}+b_{1} t^{k-1}+\ldots+b_{k}=\tilde{F}(t, b) .
$$

Arnol'd's condition for our projection $\pi$ is now equivalent to $\frac{\partial \psi_{k}}{\partial b_{1}}(0) \neq 0$, where $\psi_{k}$ is 
the $u$-coordinate of $\psi$. From the chain rule we find that

$$
\frac{\partial \tilde{G}}{\partial t}(\phi(t, 0), 0) \frac{\partial \phi}{\partial b_{j}}(t, 0)+\sum \frac{\partial \tilde{G}}{\partial a_{i}}(\phi(t, 0), 0) \frac{\partial \psi_{i}}{\partial b_{j}}(0)+\frac{\partial \tilde{G}}{\partial u}(\phi(t, 0), 0) \frac{\partial \psi_{k}}{\partial b_{j}}(0)=t^{k-i}
$$
Since $\tilde{G}$ is an unfolding of an $A_{k}$ singularity $\frac{\partial \tilde{G}}{\partial t}(\phi(t, 0), 0)$ has a Taylor series starting
with terms of degree at least $k$. So

$$
\sum \frac{\partial \tilde{G}}{\partial a_{i}}(\phi(t, 0), 0) \frac{\partial \psi_{i}}{\partial b_{j}}(0)+\frac{\partial \tilde{G}}{\partial u}(\phi(t, 0), 0) \frac{\partial \psi_{k}}{\partial b_{j}}(0) \equiv t^{k-i} \bmod \left\langle t^{k}\right\rangle
$$

If

$$
\frac{\partial \tilde{G}}{\partial a_{i}}(\phi(t, 0), 0) \equiv \sum_{1}^{k} a_{\mathrm{p} i} \mathrm{p}^{\mathrm{p}-1} \bmod \left\langle t^{k}\right\rangle \text { and } \frac{\partial \tilde{G}}{\partial u}(\phi(t, 0), 0) \equiv \sum_{1}^{k} a_{\mathrm{pk}} t^{\mathrm{p}-1} \bmod \left\langle t^{k}\right\rangle
$$

then clearly

$$
\left(a_{p i}\right)\left(\frac{\partial \psi_{i}}{\partial b_{j}}(0)\right)=\left(\delta_{p-1 k-j}\right)
$$

where $\delta$ is the usual Kronecker symbol. Using the cofactor method of inverting matrices it is not hard to see that $\frac{\partial \psi_{k}}{\partial b_{1}} \neq 0$ if and only if $\operatorname{det}\left(a_{\mathrm{p} i}\right) \neq 0$, where $1 \leq p, i \leq k-1$.

In our case where we are considering the unfolding $G$ one easily checks that this latter condition is equivalent to the condition given in (b) above.

In the case of the bifurcation set one notes that if $H$ is a versal unfolding of a potential function germ $h$ which an $A_{k+1}$ singularity then $\frac{\partial H}{\partial t}$ is a versal unfolding of the function germ $\frac{d h}{d t}$, which has an $A_{k}$ singularity, and the discriminant set of $\frac{\partial H}{\partial t}$ coincides with the bifurcation set of $H$. The condition then for the projection $\pi$ above to be generic is that $\frac{\partial^{2} G}{\partial a_{i} \partial t}(t, 0,0)(1 \leq i \leq k-1)$ should span $\mathbf{R}[t] /\left\langle t^{k-2}\right\rangle$ which clearly coincides with the condition given in (b).

Note. The Morse components of the projection, which Arnol'd discusses in [1], never arise in this paper.

2. Plane curves. The situation we shall be considering is as follows. If $U$ is the interval $(-1,2)$ and $C$ a circle we shall consider isotopies $\Phi: C \times U \rightarrow \mathbf{R}^{2}, \Phi(t, u)=\Phi_{u}(t)$, which we shall regard as a smooth isotopy between $\Phi_{0}(C)=C_{0}$ and $\Phi_{1}(C)=C_{1}$. We shall always suppose that $C_{0}$ and $C_{1}$ are generic (the precise meaning of generic will depend on what features of the curves $C_{t}$ we are considering.) Our wish is to replace the isotopy $\Phi$ by one which is generic in that projecting the relevant bifurcation or discriminant sets onto $U$ yields a generic function on these sets in the sense of Arnol'd. We shall first review the 
geometry associated with a single generic curve and then compute the conditions for an isotopy to be generic. In $\$ 4$ we shall prove the relevant transversality lemma which will ensure that the conditions are generically satisfied.

I. The Height Functions. First we shall consider the geometry of plane curves reflected in their contact with lines. So if $S$ is the unit circle in $\mathbf{R}^{2}$ we are considering the family of height functions parametrized by $S$.

(a) Single curve. Given a smooth curve $\phi: C \rightarrow \mathbf{R}^{2}$ we have a family of height functions $h: C \times S \rightarrow \mathbf{R}$ defined by $h(t, a)=\phi(t), a=h_{a}(t)$ (where . denotes the usual inner product in $\left.\mathbf{R}^{2}\right)$. The set of pairs

$$
\boldsymbol{\sigma}=\left\{(t, a): h_{a}: C \rightarrow \mathbf{R} \text { has an } A_{l} \text { singularity at } t \text { for some } l \geq 1\right\}
$$

is the unit normal bundle to $C$. If $\sigma^{+}$is one of the two components of $\sigma$, the set $\delta=\left\{(h(t, a), a) \in \mathbf{R} \times S:(t, a) \in \sigma^{+}\right\}$is the affine dual of $C$. The natural projection $p: \sigma \rightarrow S$ is the Gauss map of $C$. (See [3] for details.)

Ignoring quasi-global phenomena, one has the following results. Generically the $h_{a}$ have only $A_{1}$ and $A_{2}$ singularities, both versally unfolded by the family $h$. The $A_{2}$ singularities correspond to ordinary inflexions of the curve. The corresponding points on the affine dual are cusp points, and the Gauss map has ordinary folds associated with each inflexion.

(b) An isotopy of curves. Let $\Phi: C \times U \rightarrow \mathbf{R}^{2}$ be an isotopy as above, and consider the associated family of height functions

$$
H: C \times U \times S \rightarrow \mathbf{R}, H(t, u, a)=\Phi(t, u) . a=H_{a}(t, u) .
$$

The set $\Sigma=\left\{(t, u, a): H_{a}(-, u): C \times\{u\} \rightarrow \mathbf{R}\right.$ has an $A_{l}$ singularity at $t$ for some $\left.l \geq 1\right\}$ is the family of unit normal bundles. If $\Sigma^{+}$is one of the two components of $\Sigma$ the set $\Delta=\left\{(H(t, u, a), u, a) \in \mathbf{R} \times U \times S:(t, u, a) \in \Sigma^{+}\right\}$is the family of affine duals. The natural projection $P: \Sigma \rightarrow U \times S$ is the family of Gauss maps.

The set $\Delta$ has the structure of a discriminant set, and the set of critical values of $P$ the structure of a bifurcation set. We now consider conditions under which these sets are generic and their natural projections to $U$ also are.

(i) The duals. Without loss of generality we shall work at $u=0$ and $t=0$. We write $\Phi(t, 0)=\left(t, c_{2} t^{2}+c_{3} t^{3}+c_{4} t^{4}+O(5)\right)$ where $O(k)$ denotes a smooth function on $\mathbf{R}$ vanishing at $t=0$ to order $k-1$. If $a=(0,1)$ the condition for $h_{a}$ to have a singularity of type $A_{l}$ for some $l \geqslant k$ at $t=0$ is $c_{2}=\cdots=c_{k}=0$. Generically we expect $A_{1}$ and $A_{2}$ singularities of the height functions on the $C_{u}$, with $A_{3}$ singularities occuring for isolated values of $u$. Clearly from the unfolding parameter $a$ we obtain $\frac{\partial H_{a}}{\partial a}(t, 0)=t$. So we can express the condition for a generic projection in terms of

$$
\psi=\frac{\partial H_{a}}{\partial u}(t, 0)=\frac{\partial \Phi_{2}}{\partial u}(t, 0)=d_{1} t+d_{2} t^{2}+O(3) \quad \text { say. }
$$

(ii) $A_{1}$ and $A_{2}$. Here since $1, \frac{\partial H_{a}}{\partial a}(t, 0)=t$ span $\mathbf{R}[t] /\left\langle t^{k}\right\rangle$ by $1.2($ a), the projection is the trivial one. 
(iii) $A_{3}$. The condition for a versal unfolding is that $1, t, \psi$ span $\mathbf{R}[t] /\left\langle t^{3}\right\rangle$ i.e. $d_{2} \neq 0$. The condition that the projection is then generic (in $1.2(\mathrm{~b})$ ) is that in addition $1, \frac{\partial H_{a}}{\partial a}(t, 0)$
span $\left.\mathbf{R}[t] / t^{2}\right\rangle$, which is automatic.

(iv) Singularities of the Gauss map. As explained in $\S 1$ the conditions for the bifurcation sets to be generic with the projection to $u$ generic coincide with those given above.

II. Distance Squared Functions. We now consider the geometry of plane curves reflected in their contact with circles. Thus we are considering the family of distance squared functions from points of $\mathbf{R}^{2}$ parametrized by $\mathbf{R}^{2}$.

(a) Single Curve. Given a smooth curve $\phi: C \rightarrow \mathbf{R}^{2}$ we have a family of distance squared functions $d: C \times \mathbf{R}^{2} \rightarrow \mathbf{R}$ defined by $d(t, a)=\|\phi(t)-a\|^{2}=d_{a}(t)$ (where $\|$.$\| is the$ Euclidean norm on $\mathbf{R}^{2}$ ). The set of pairs

$$
\sigma=\left\{(t, a): d_{a}: C \rightarrow \mathbf{R} \text { has an } A_{l} \text { singularity at } t \text { for some } l \geq 1\right\}
$$

is the normal bundle to $C$. The natural projection $p: \sigma \rightarrow \mathbf{R}^{2}$ is the exponential mapping of the normal bundle and the set of critical values of $p$ is the evolute of $C$. (See [6] for details.)

Generically the $d_{a}$ have only $A_{1}, A_{2}$ and $A_{3}$ singularities, all versally unfolded by the family $d$. The function $d_{a}$ has an $A_{l}$ singularity (where $l \geq 2$ ) at $t$ if and only if $a$ is the centre of curvature of $C$ at $\phi(t)$. The function $d_{a}$ is of type $A_{l}$ (where $l \geq 3$ ) if, in addition, $\phi(t)$ is a vertex of $C$. The point on the evolute corresponding to a vertex is a cusp point.

(b) An isotopy of curves. Let $\Phi$ be as above and consider $D: C \times U \times \mathbf{R}^{2} \rightarrow \mathbf{R}$, $D(t, u, a)=\|\Phi(t, u)-a\|^{2}=D_{a}(t, u)$. The set

$$
\Sigma=\left\{(t, u, a): D_{a}(-, u): C \times\{u\} \rightarrow \mathbf{R} \text { has an } A_{l} \text { singularity at } t \text { for some } l \geqslant 1\right\}
$$

is the family of normal bundles. The natural projection $P: \Sigma \rightarrow U \times \mathbf{R}^{2}$ is the family of exponential maps. The set of critical values of $P$ is the family of evolutes. This set has the structure of a bifurcation set; we seek conditions under which it is generic and so is its natural projection onto $U$.

Generically we expect $A_{1}, A_{2}$ and $A_{3}$ singularities of the distance squared functions on the $C_{u}$, with $A_{4}$ singularities occuring for isolated values of $u$. Without loss of generality we shall work at $u=0$ and $t=0$. As before we write $\Phi(t, 0)=$ $\left(t, c_{2} t^{2}+c_{3} t^{3}+c_{4} t^{4}+O(5)\right)$. If $b=\left(0,1 /\left(2 c_{2}\right)\right)$ the condition for $d_{b}$ to have a singularity of type $A_{k}$ is as follows.

$d_{b}$ is of type $A_{2}$ if and only if $c_{3} \neq 0$.

$d_{b}$ is of type $A_{3}$ if and only if $c_{3}=0, c_{4}-c_{2}^{3} \neq 0$.

$d_{b}$ is of type $A_{4}$ if and only if $c_{3}=c_{4}-c_{2}^{3}=0, c_{5} \neq 0$.

$d_{b}$ is of type $A_{l}(l \geqslant 5)$ if and only if $c_{3}=c_{4}-c_{2}^{3}=0, c_{5}=0$.

Clearly from the tangent space to $\mathbf{R}^{2}$ we obtain $t$ and $\left(-1 /\left(2 c_{2}\right)+c_{2} t^{2}+c_{3} t^{3}+c_{4} t^{4}+O(5)\right)$. What about the tangent space to $U$ ? 
The partial derivative

$$
\left.\frac{\partial D}{\partial u} b(t, u)\right|_{u=0}=0=2\left(\Phi_{1}(t, 0) \frac{\partial \Phi_{1}}{\partial u}(t, 0)+\Phi_{2}(t, 0) \frac{\partial \Phi_{2}}{\partial u}(t, 0)-\rho \frac{\partial \Phi_{2}}{\partial u}(t, 0)\right)
$$

where $\rho=1 /\left(2 c_{2}\right)$. Setting

$$
\frac{\partial \Phi_{1}}{\partial u}(t, 0)=d_{0}+d_{1} t+d_{2} t^{2}+O(3), \quad \frac{\partial \Phi_{2}}{\partial u}(t, 0)=e_{0}+e_{1} t+e_{2} t^{2}+e_{3} t^{3}+O(4)
$$

clearly this derivative is

$$
2\left(t\left(d_{0}+d_{1} t+d_{2} t^{2}\right)+\left(c_{2} t^{2}+c_{3} t^{3}\right)\left(e_{0}+e_{1} t\right)-\rho\left(e_{0}+e_{1} t+e_{2} t^{2}+e_{3} t^{3}\right)+O(4)\right)
$$

i.e. $2\left(-\rho e_{0}+t\left(d_{0}-\rho e_{1}\right)+t^{2}\left(d_{1}+c_{2} e_{0}-\rho e_{2}\right)+t^{3}\left(d_{2}+c_{2} e_{1}+c_{3} e_{0}-\rho e_{3}\right)+O(4)\right)$.

(i) $A_{1}, A_{2}$ and $A_{3}$. Here $1, t$ and $\left(-\beta+c_{2} t^{2}+\cdots\right)$ span $\mathbf{R}[t] /\left\langle t^{k}\right\rangle$. So the projection of the family of evolutes is trivial.

(ii) $A_{4}$. First we need $1, t,\left(-\beta+c_{2} t^{2}+c_{3} t^{3}\right)$ and $\left(^{*}\right)$ to span $\mathbf{R}[t] /\left\langle t^{4}\right\rangle$. Using $c_{3}=$ $c_{4}-c_{2}^{3}=0$ one finds that this is so if and only if $c_{2}\left(d_{2}+c_{2} e_{1}-\rho e_{3}\right)=c_{2} d_{2}+c_{2}^{2} e_{1}-\frac{1}{2} e_{3} \neq 0$. For the projection to be generic in addition we require $1, t$ and $-\beta+c_{2} t^{2}$ to span $\mathbf{R}[t] /\left\langle t^{3}\right\rangle$ which they clearly do always.

3. The transversality results. One approach to the generic geometry of plane curves (discussed elsewhere by the author) goes as follows. Given a smooth plane curve $C$ with an orientation, at each point $p$ of $C$ choose the positive tangent and outward normal as $x$ and $y$ axes. The curve near $p$ then has a unique representation as the graph $y=f(x)$, with $f^{\prime}(0)=0$. If $V_{\mathrm{p}}^{\mathrm{k}}$ is the vector space spanned by monomials of degree $d$ in one variable $x$, with $p \leq d \leq k$, taking the truncated Taylor expansion of $f$ (to degree $k$ ) gives a smooth map $\gamma: C \rightarrow V_{2}^{k}$. There are then certain algebraic subsets of $V_{2}^{k}$ of codimension at least 2 which one wants to avoid in order to ensure that, for example, the height functions are generic on $C$. Thus one needs to prove one can make $\gamma$ transverse to (and hence miss) such sets. We need to generalise this type of result.

When we concern ourselves with isotopies then it is clear from our work in $\$ 2$ that we need to consider the components of $\frac{\partial \Phi}{\partial u}(t, u(0))$ in the tangent and normal directions to $C_{u(0)}$ at $\Phi(t, u(0))$. Thus for each $k(k \geq 2)$ we consider a map $\Gamma: C \rightarrow V_{2}^{k} \times V_{0}^{k} \times V_{0}^{k}$. The first component of $\Gamma$ is $\gamma$. The second and third components of $\Gamma$ are defined as follows.

By a change of $t$ co-ordinates we may suppose that with respect to the $x$ and $y$ axes as above $\Phi(t, u(0))=(t, f(t))$ for some smooth $f$. The second and third components of $\Gamma$ are the Taylor expansions of $\left.\frac{\partial \Phi_{1}}{\partial s}(t, u(0)+s)\right|_{s=0},\left.\frac{\partial \Phi_{2}}{\partial s}(t, u(0)+s)\right|_{s=0}$ with respect to the above coordinate systems, truncated to degree $k$.

Clearly we shall want to obtain a transversality theorem for the map $\Gamma$. To do this we proceed as follows. Given an isotopy $\Phi: C \times U \rightarrow \mathbf{R}^{2}$ as above between $C_{0}$ and $C_{1}$ then shrinking $U$ (to an open neighbourhood of $[0,1]$ ) we may suppose that $\Phi(C \times U)$ is 
contained in some large ball neighbourhood of the origin $B \subset \mathbf{R}^{2}$. Let $P$ denote the space of polynomial maps of degree at most $d$ from $\mathbf{R}^{2}$ to itself, and choose a sufficiently small convex neighbourhood $W$ of the zero map in $P$ so that for any $\psi_{1}, \psi_{2}$ in $W, I+\psi_{1}+\psi_{2}$ maps the ball $2 B$ of twice the radius of $B$ diffeomorphically onto its image (where $I$ is the identity). Now consider $\tilde{\Phi}: C \times U \times W^{2} \rightarrow \mathbf{R}^{2}$ defined by $\tilde{\Phi}\left(t, u, \psi_{1}, \psi_{2}\right)=\Phi(t, u)+$ $\psi_{1}(\Phi(t, u))+u \psi_{2}(\Phi(t, u))$. For each $u, \psi_{1}, \psi_{2}, t \rightarrow \tilde{\Phi}\left(t, u, \psi_{1}, \psi_{2}\right)$ is an embedding of $C$ in $\mathbf{R}^{2}$. There is clearly an associated mapping $\tilde{\Gamma}: C \times U \times W^{2} \rightarrow V_{2}^{k} \times V_{0}^{k} \times V_{0}^{k}$.

LemMA 3.1. The map $\tilde{\Gamma}$ is a submersion at $(t, 0,0,0)$.

Proof. We may as well work at $(0,0)$. Consider $\psi_{1}=\left(0, s x^{r}\right), \psi_{2}=0$ (for $s$ small and nonzero) and the quotient

$$
\left(\tilde{\Gamma}\left(t, 0, \psi_{1}, \psi_{2}\right)-\tilde{\Gamma}(t, 0,0,0)\right) s^{-1}
$$

for $r \geq 2$. Writing $\Phi(t, u)=\left(\Phi_{1}(t, u), \Phi_{2}(t, u)\right)$ with respect to the canonical axes at $\Phi(t, 0)$ we have

$$
\Phi\left(t, u, \psi_{1}, \psi_{2}\right)=\Phi(t, u)+\left(0, s\left(\Phi_{1}(t, u)\right)^{r}\right)
$$

Hence at $u=0$ we get $\left(t, \Phi_{2}(t, 0)+s t^{r}\right)$ and the limit of the quotient above as $s \rightarrow 0$ has $x^{r}$ in its first component. It is irrelevant what the second and third components are although they are easily computed.

Now consider $\psi_{1}=0, \psi_{2}=\left(s x^{r}, 0\right)$ (resp. $\psi_{2}=\left(0, s x^{r}\right)$ ) for $s$ small and nonzero, and the corresponding quotient $\left({ }^{*}\right)$ above. Here we have

$$
\begin{gathered}
\tilde{\Phi}\left(t, u, \psi_{1}, \psi_{2}\right)=\Phi(t, u)+u\left(s\left(\Phi_{1}(t, u)\right)^{r}, 0\right) \\
\left(\operatorname{resp} . \tilde{\Phi}\left(t, u, \psi_{1}, \psi_{2}\right)=\Phi(t, u)+u\left(0, s\left(\Phi_{1}(t, u)\right)^{r}\right)\right) .
\end{gathered}
$$

Clearly when $u=0$ we obtain $\Phi(t, 0)$ and the first component of the limit of $\left(^{*}\right)$ as $s \rightarrow 0$ is 0 in both cases. To determine the second two we note that differentiating $\bar{\Phi}$ with respect to $u$ in the tangent and normal directions at $\Phi(t, 0)$ and setting $u=0$ we obtain

$$
\frac{\partial \Phi_{1}}{\partial u}(t, 0)+s\left(\Phi_{1}(t, 0)\right)^{r}=\frac{\partial \Phi_{1}}{\partial u}(t, 0)+s t^{r} \text { and } \frac{\partial \Phi_{2}}{\partial u}(t, 0) \quad\left(\text { resp. } \frac{\partial \Phi_{1}}{\partial u}(t, 0) \text { and } \frac{\partial \Phi_{2}}{\partial u}(t, 0)+s t^{r}\right) \text {. }
$$

And hence the limit of $\left(^{*}\right)$ as $s \rightarrow 0$ is $\left(0, x^{r}, 0\right)$ (resp. $\left.\left(0,0, x^{r}\right)\right)$ and our assertion is proved.

Of course as it stands the lemma is of little use since it only gives us information near $u=0$. Had we considered the family $\Phi(t, u)+\psi_{1}(\Phi(t, u))+(u-\alpha) \psi_{2}(\Phi(t, u))$ we would have had some control over the behaviour of the isotopy near $u=\alpha$ instead. Our idea is to try to glue together deformations of the isotopy obtained by taking sufficiently many $\alpha$ 's in $[0,1]$. To do this we proceed as follows.

Consider the map $C \times U \times W^{2} \times U \rightarrow \mathbf{R}^{2}$ defined by

$$
\left(t, u, \psi_{1}, \psi_{2}, \alpha\right) \mapsto \Phi(t, u)+\psi_{1}(\Phi(t, u))+(u-\alpha) \psi_{2}(\Phi(t, u))
$$

This yields a corresponding map $\Gamma^{*}$, obtained from $\Gamma$ by fixing $\alpha, \psi_{1}, \psi_{2}$, which is a submersion at each point $(t, u, 0,0, u)$ by the lemma above. Using the fact that we are only interested in the isotopy for $u \in[0,1] \subset U$ we can find neighbourhoods $U_{1}$ of $[0,1]$, 
$W_{1}$ of $0 \in W$, and $\varepsilon>0$ such that, for $u \in U_{1}, \psi_{j} \in W$, and $|u-\alpha|<\varepsilon$, the map $\Gamma^{*}$ is a submersion at $\left(t, u, \psi_{1}, \psi_{2}, \alpha\right)$. Now choose equally spaced $\alpha_{1}, \alpha_{2}, \ldots, \alpha_{N}$ with $0=\alpha_{1}<\alpha_{2}<\ldots<\alpha_{N}=1$ and $\left|\alpha_{j+1}-\alpha_{j}\right|<\varepsilon / 2$, and for each $\alpha_{j}$ consider the map $\Gamma_{j}^{*}$ obtained from $\Gamma^{*}$ by fixing $\alpha=\alpha_{i}$, and replacing $W$ by $W_{1}$ and $U$ by $U_{1}$.

We now obtain the transversality result in the usual way for each $\Gamma_{j}^{*}$ by applying Thom's lemma [5, p. 49]. So given any Whitney (A) regular stratified set $X$ in $V_{2}^{k} \times V_{0}^{k} \times V_{0}^{k}$ we can find arbitrarily small $\psi_{1}, \psi_{2}$ with the isotopy

$$
(t, u) \mapsto \Phi(t, u)+\psi_{1}(\Phi(t, u))+\left(u-\alpha_{j}\right) \psi_{2}(\Phi(t, u))
$$

yielding $\Gamma_{i}^{*}\left(\psi_{1}, \psi_{2}\right): C \times U_{1} \rightarrow V_{2}^{k} \times V_{0}^{k} \times V_{0}^{k}$ which is transverse to $X$, provided that $\left|u-\alpha_{i}\right|<\varepsilon$. Indeed we can clearly do more. We can also ensure that when $u=\alpha_{j+1}$ or $u=\alpha_{j-1}$ the corresponding curve is generic for height and/or distance squared functions. Moreover since the set of $\left(\psi_{1}, \psi_{2}\right)$ excluded by an application of Thom's lemma is of measure zero, we can choose a fixed $\left(\psi_{1}, \psi_{2}\right)$ so that $\Gamma_{i}^{*}\left(\psi_{1}, \psi_{2}\right)$ and $\Gamma_{j}^{*}\left(\psi_{1},-\psi_{2}\right)$ are transverse to $X$, for all $j$, provided again that $\left|u-\alpha_{j}\right|<\varepsilon$, and that $u=\alpha_{j+1}$ or $u=\alpha_{j-1}$ gives generic curves.

But these individual isotopies can now be glued together because

$$
\begin{aligned}
& \Phi\left(t, \alpha_{j+1}\right)+\psi_{1}\left(\Phi\left(t, \alpha_{i+1}\right)\right) \pm\left(\alpha_{j+1}-\alpha_{j}\right) \psi_{2}\left(\Phi\left(t, \alpha_{j+1}\right)\right) \\
= & \Phi\left(t, \alpha_{i+1}\right)+\psi_{1}\left(\Phi\left(t, \alpha_{i+1}\right)\right) \mp\left(\alpha_{j+1}-\alpha_{j+2}\right) \psi_{2}\left(\Phi\left(t, \alpha_{j+1}\right)\right)
\end{aligned}
$$

and $\left|\alpha_{i}-\alpha_{i+1}\right|<\varepsilon$ so that we can piece together the generic isotopies obtained from fixing $\alpha=\alpha_{1}, \alpha_{3}, \alpha_{5}, \ldots$. Of course glueing the isotopies together is no problem since the curves at the endpoints of the relevant intervals are generic. Now this does not yield a generic isotopy from $C_{0}$ to $C_{1}$ but these curves are themselves generic, so provided $\psi_{1}$ and $\psi_{2}$ are sufficiently small the end curves of our generic isotopy will be isotopic through a family of generic curves to $C_{0}$ and $C_{1}$. (This uses the fact that genericity is open.) So we have proved the following result.

THEOREM 3.2. Let $X$ be a Whitney (A) regular stratified subset of $V_{2}^{k} \times V_{0}^{k} \times V_{0}^{k}$. If $\Phi(t, u)$ is an isotopy between the generic curves $C_{0}$ and $C_{1}$ then it can be arbitrarily closely approximated by an isotopy $\theta(t, u)$ with $\theta(t, 0)=\Phi(t, 0), \theta(t, 1)=\Phi(t, 1)$, and the corresponding $\Gamma$ transverse to $X$.

Thus we now seek the stratified set $X$.

I. Height Functions. Take the strata to be (a) $0 \times V_{0}^{4} \times V_{0}^{4}(k=4)$ to ensure $A_{l}, l \leqslant 3$, (b) $0 \times\left\{d_{1} x+d_{3} x^{2}\right\} \times V_{0}^{3}(k=3)$ to ensure that the projection at an $A_{3}$ point is generic.

II. Distance Squared Functions. Take the strata to be

(a) $\left\{c_{2} x^{2}+c_{2}^{3} x^{4}\right\} \times V_{0}^{5} \times V_{0}^{5}(k=5)$ to ensure $A_{l}, l \leq 4$,

(b) $\left\{c_{2} x^{2}+c_{2}^{3} x^{4}+c_{5} x^{5}\right\} \times V_{0}^{5} \times\left\{e_{0}+e_{1} x+e_{2} x^{2}+2\left(c_{2} d_{2}+c_{2}^{2} e_{1}\right) x^{3}+e_{4} x^{4}+e_{5} x^{5}\right\}(k=5)$

to ensure the projection at the $A_{4}$ point is generic.

In both cases the strata are clearly of codimension at least 3 , and hence the $\tilde{\Gamma}_{\left(\psi_{1}, \psi_{2}\right)}$ is transverse to $X$ if and only if it misses $X$, which ensures that the isotopy $\tilde{\Phi}_{\left(\psi_{1}, \psi_{2}\right)}$ is generic. 


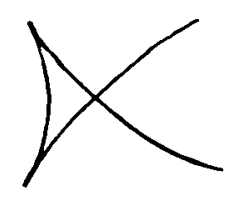

(i)

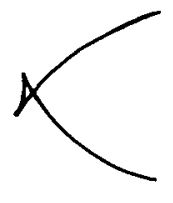

(ii)

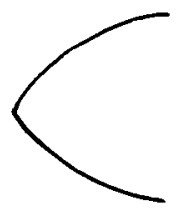

(iii)

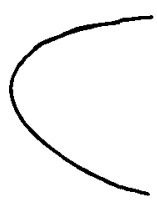

(iv)

Figure 1

\section{The pictures.}

I. Height Functions.

(i) The duals. As always ignoring quasi-global phenomena there is only one "catastrophic" event in a generic isotopy and this occurs when the height function acquires an $A_{3}$ singularity. Thus a vertex and a parabolic point (i.e. point of zero curvature) coincide. The duals are locally given by generic slices of the swallowtail and undergo the changes drawn in Fig. 1, the catastrophic event taking place at stage (iii). Thus the event can be viewed as the birth (or annihilation) of two parabolic points, together with the birth (or death) of a local bitangent. The corresponding curve one expects to be modelled (locally) by the one parameter family of curves $y=x^{4}+u x^{2}$. One can clearly see the way in which the inflexions are destroyed and the bitangent disappears as $u$ increases through 0 , with the normal height function at the origin, when $u=0$, being of type $A_{3}$ (see Fig. 2). Note that since any plane curve is isotopic to a circle (with no inflexions) every generic curve has an even number of inflexions.

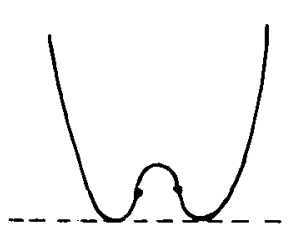

(i) $u<0$

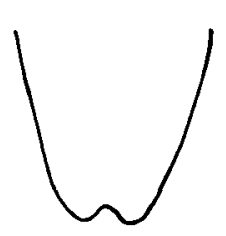

(ii) $u<0$

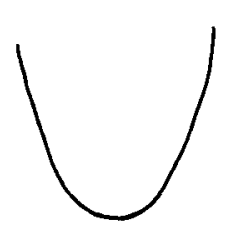

(iii) $u=0$

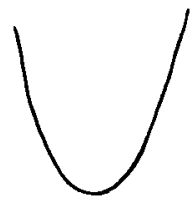

(iv) $u<0$

Figure 2

(ii) The Gauss maps. The corresponding changes in the Gauss map, or rather the critical values of the Gauss map which these results give, are not as interesting. They are given by generic slices of the cusp (Fig. 3) and again we have the birth or annihilation of two critical values (and points) of the Gauss map. Using the model $y=x^{4}+u x^{2}$ again one can view the family of Gauss maps as the projections of the usual folded surface associated with the cusp catastrophe with the parameter $u$ being in the cuspidal tangent direction (Fig. 4). So as $u$ increases through zero one can think of the $s$-shaped curves which are flattened onto the unit circle $S$ sliding over each other until the twofold points spring out simultaneously when $u=0$. We should stress however that although the model $y=x^{4}+u x^{2}$ almost certainly does describe the local generic changes of the Gauss map at an $A_{4}$ point our results only make assertions about the changes in the critical values. 


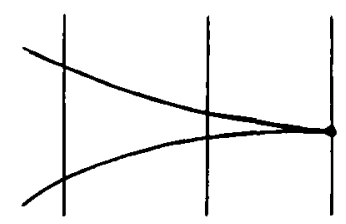

(i)

(ii)

(iii)

(iv)

Figure 3

Although this is clearly a great weakness of our approach the problem is that in more complicated situations if we want to consider all of the geometry one runs into smooth moduli, which makes the corresponding theory quite impossible. (See [4] for a more detailed discussion of this point.)

II. The Distance Squared Functions. Evolutes. Again there is only one "catastrophic" even there occurring with the acquisition of an $A_{4}$ singularity. The evolute undergoes the same changes as the dual did, the interpretation this time being the birth (or annihilation) of two vertices via a higher vertex, together with the birth (or death) of a double centre of curvature. (One can find a model for these changes (locally), namely the one parameter family of curves $y=u x+x^{2}+x^{4}+x^{5}$.) Again since any generic curve is isotopic through a generic family to say an ellipse (with four vertices), clearly every generic curve has an even number of vertices. (Although this is a trivial observation the same idea yields less trivial information in higher dimensions.)
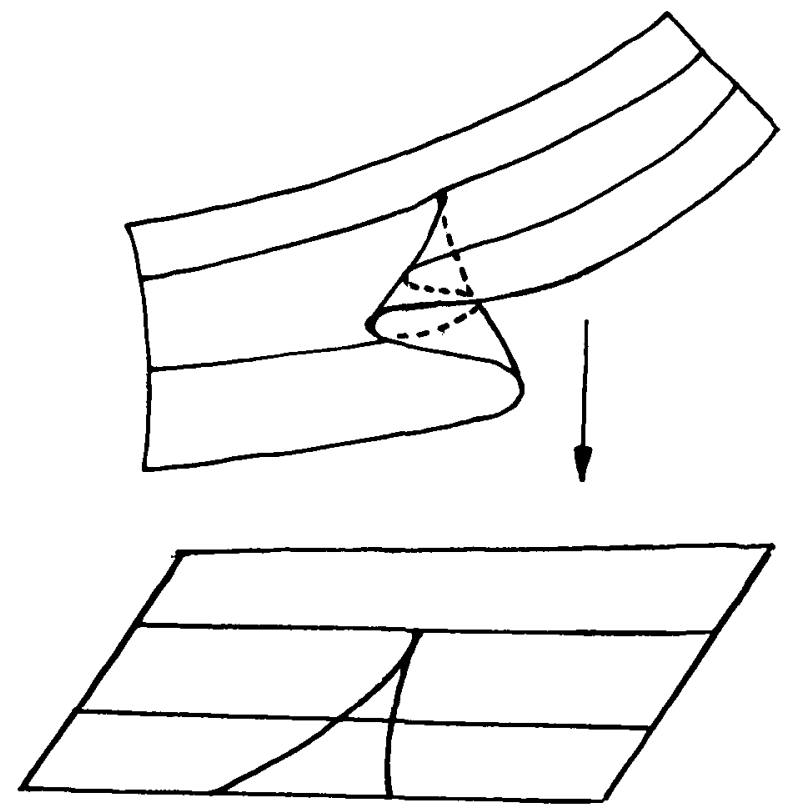

Figure 4 


\section{REFERENCES}

1. V. I. Arnol'd, Wavefront evolution and equivariant Morse lemma, Comm. Pure Appl. Math. 29 (1976), 557-582.

2. Th. Brocker and L. Lander, Differentiable germs and catastrophes, London Math. Soc. Lecture Note Series, No. 17 (Cambridge University Press, 1975).

3. J. W. Bruce, The duals of generic hypersurfaces, Math. Scand. 49 (1981), 36-60.

4. J. W. Bruce, Generic functions on algebraic sets, to appear.

5. C. G. Gibson, Singular points of smooth mappings, Pitman Research Notes in Mathematics, 25 (Pitman, 1979).

6. I. R. Porteous. The normal singularities of a submanifold, J. Differential Geometry 5 (1971), 543-564.

UnIVERSITY COLlege,

CORK,

IRELAND. 